\title{
Bonde universitário: ações de prevenção das infecções sexualmente transmissíveis: um relato de experiência
}

\author{
University tram: prevent actions sexually transmitted infections: an experience report. \\ Tranvía universitaria: acciones para prevenir las infecciones de transmisión sexual: um \\ informe de experiencia.
}

Lilian Conceição Guimarães de Almeida ${ }^{1 *}$, Jamile Mendes da Silva Santos ${ }^{1}$, Yone Cardeal da Silva $^{1}$, Rebeca Nascimento dos Santos Mascarenhas ${ }^{1}$, Maiara Oliveira dos Santos ${ }^{1}$, Daine Ferreira Brazil do Nascimento ${ }^{1}$, Bruna Prates Lopes Brasil'.

\section{RESUMO}

Objetivo: Relatar a experiência de graduandas de enfermagem em ações extensionistas de prevenção das Infecções Sexualmente Transmissíveis (IST). Relato de experiência: O projeto é composto por graduandas de enfermagem da Universidade Federal da Bahia. Para subsidiar as ações realizadas houve a formação teórico/prática, na qual eram realizadas discussões semanais fomentando uma maior aproximação da equipe executora com a temática. As ações extensionistas foram fundamentadas a partir de metodologias ativas e realizadas nos mais distintos espaços entre ruas, escolas e campi da universidade. Durante as abordagens foram disponibilizados materiais educativos sobre IST, insumos de prevenção como preservativos e gel lubrificante, além da realização de testagem para HIV. As capacitações que antecederam as atividades oportunizaram a ampliação dos conhecimentos e trocas de experiências significativas. Foi possível identificar que muitas pessoas adotam comportamentos de invulnerabilidade. Em contrapartida, também houve devolutivas positivas como expressões de interesse, curiosidade e relatos de vivências sobre o assunto. As ações de prevenção extramuros implementadas foram percebidas como um importante mecanismo de enfrentamento das IST. Considerações finais: Falar sobre IST ainda é um tabu na sociedade. Atividades extensionistas, são fundamentais para o fortalecimento do vínculo entre academia e comunidade.

Palavras-chave: Educação em saúde, Doenças sexualmente transmissíveis, Bacharelado em enfermagem.

\begin{abstract}
Objective: To report the experience of nursing undergraduate students in extension actions for the Sexually Transmitted Infections (STI prevention. Experience report: The project is composed of nursing undergraduate students at the Federal University of Bahia. In order to subsidize the actions carried out, there was theoretical training, in which weekly discussions were held, promoting a greater approximation of the executing team with the theme. The extension actions were based on active methodologies and carried out in the most different spaces between streets, schools and university campuses. During the approaches, educational materials on STI were made available, prevention supplies such as condoms and lubricating gel, beyond addition to HIV testing. The training that preceded the activities provided an opportunity to expand knowledge and exchange significant experiences. It was possible to identify that many people adopt an invulnerability behavior. On the other hand, there were also positive feedback as expressions of interest, curiosity and reports of experiences on the subject. The extra-wall actions prevention implemented were
\end{abstract}

${ }^{1}$ Universidade Federal da Bahia (UFBA) Salvador - Bahia. *E-mail: liliancgalmeida@yahoo.com.br

SUBMETIDO EM: $3 / 2020$

ACEITO EM: 4/2020

PUBLICADO EM: 5/2020

REAS/EJCH | Vol.12(6) | e3268 | DOI: https://doi.org/10.25248/reas.e3268.2020 Página 1 de 6 
perceived as an important mechanism for coping with STI. Final considerations: Talking about STI is still taboo in society. Extension activities are essential for strengthening the link between academia and the community.

Keywords: Health education, Sexually transmitted diseases, Nursing baccalaureate.

\section{RESUMEN}

Objetivo: Informar sobre la experiencia de estudiantes de pregrado de enfermería en acciones de extensión para la prevención de infecciones de transmisión sexual (ITS). Informe de experiencia: el proyecto está compuesto por estudiantes universitarios de enfermería de la Universidad Federal del Bahía. Para subsidiar las acciones realizadas, se realizó una capacitación teórica en la cual se realizaron discusiones semanales, promoviendo una mayor aproximación del equipo ejecutor con el tema. Las acciones de extensión se basaron en metodologías activas y se llevaron a cabo en los espacios más diferentes entre calles, escuelas y campus universitarios. Durante los enfoques, se pusieron a disposición materiales educativos sobre las ITS, suministros de prevención como condones y gel lubricante, además de pruebas de VIH. La capacitación que precedió a las actividades brindó la oportunidad de expandir el conocimiento e intercambiar experiencias significativas. Fue posible identificar que muchas personas adoptan un comportamiento de invulnerabilidad. Por otro lado, también hubo comentarios positivos como expresiones de interés, curiosidad e informes de experiencias sobre el tema. Las acciones de prevención extramuros implementadas se percibieron como un mecanismo importante para hacer frente a las ITS. Consideraciones finales: Hablar sobre las ITS sigue siendo un tabú en la sociedad. Las actividades de extensión son esenciales para fortalecer el vínculo entre la academia y la comunidad.

Palabras clave: Educación en salud, Enfermedades de transmisión sexual, Bachillerato en enfermería.

\section{INTRODUÇÃO}

As Infecções Sexualmente Transmissíveis (IST) são manifestações causadas por vírus, bactérias ou outros microrganismos, disseminadas, principalmente, por meio do contato sexual (oral, vaginal e/ou anal) sem o uso de preservativo vaginal ou peniano, com um indivíduo previamente infectado (BRASIL, 2019). A adesão ao uso de preservativos é a principal estratégia de prevenção das IST, mas não a única (BRASIL, 2015). Vale ressaltar, que já são identificados muitos avanços no que se refere ao enfrentamento da disseminação dessas infecções, outras iniciativas precisaram ser pensadas para conter a transmissibilidade da epidemia. Hoje, no que se refere ao HIV especificamente, fala-se em ações de prevenção que incluam estratégias combinadas, uso simultâneo de medidas como medicamentos antirretrovirais, testagem sorológicas, incentivos para mudança de comportamento, redução de danos, entre outras (BRASIL, 2019).

Mas, a prevenção das IST é uma tarefa árdua, que envolve questões de ordens estruturais, sociais, comportamentais que estão arraigadas na sociedade, inclui também as dificuldades de acesso aos serviços, preconceito, estigmas e estereótipos que limitam a população a ter informação, conhecimento para implementar práticas sexuais seguras (BRASIL, 2017). Assim, é de suma importância que hajam medidas efetivas para controle, tratamento e enfrentamento das IST. É preciso diversificar as ações de cuidado, ir além de atendimentos em serviços de saúde, ampliar a atuação para outros espaços e promover a discussão sobre esta temática. As atividades de educação em saúde podem ser excelentes ferramentas para o intercâmbio de saberes e promover o acesso à informação por meio de metodologias ativas, pode produzir reflexões, mudanças de comportamentos e favorecer uma melhor qualidade de vida para a população (PASSOS T et al, 2017).

Diante dessa perspectiva, Freire $P$ (1987) sugere que toda ação educativa promove uma troca de conhecimentos, visto que a medida em que se ensina se aprende e vice-versa. $E$ foi com base nesta ideologia que o projeto de extensão foi pensado e suas ações estruturadas, pois acreditamos que o conhecimento é algo que se constrói coletivamente. 
O Bonde Universitário: Participação jovem na prevenção de IST, vírus da imunodeficiência humana (HIV), síndrome de imunodeficiência adquirida (AIDS) e Hepatites virais, foi um projeto elaborado em novembro de 2017, que surge a partir da inquietude de uma docente e graduandas em enfermagem frente ao aumento das IST entre o público jovem, além disso, com um intuito de tornar o Bonde um dispositivo social para enfrentamento da epidemia. Baseado nesse contexto, o artigo tem por objetivo relatar a experiência de graduandas de enfermagem em ações extensionistas de prevenção das IST.

\section{RELATO DE EXPERIÊNCIA}

O projeto teve como ponto de partida a composição de uma equipe executora, com formação teórica subsidiada, discussões e compartilhamento de referências fundamentais para aprimorar e estimular a construção de conhecimento sobre a temática e sobretudo, por possibilitar a compreensão das subjetividades que envolvem o público alvo das futuras ações.

Para iniciar as discussões teóricas, foi imprescindível aproximar-se de conceitos como saúde, uma vez que iríamos falar de agravos que em sua maioria não ocasionavam problemas aparentes, mas necessitavam de medidas efetivas para prevenção, diagnóstico, tratamento e cura. Ao longo do processo de imersão teórica, outras temáticas foram sendo requeridas e aos poucos o arcabouço teórico era construído.

Para concepção teórica houve a participação de profissionais dos serviços parceiros, entre eles, enfermeiros, terapeutas ocupacionais e fisioterapeutas, que abordaram especificamente sobre as patologias (IST, HIV/Aids e hepatites) que mais acometem as pessoas atendidas no serviço e relataram suas experiências práticas nos atendimentos, incluindo aconselhamento e manejo de IST.

Por conseguinte, deu-se início ao planejamento das ações extensionistas. Nesta etapa, foram realizadas reuniões semanais, discussões e estudos sobre os temas fomentando a construção das estratégias a serem utilizadas em cada ação.

Atrelado a isso, o grupo de apoio e acolhimento de pessoas convivendo com o HIV/Aids ajudou muito nesse processo ao permitir a aproximação com um universo até então desconhecido, o lugar de fala de pessoas que convivem com o HIV. Eles discutiam as estratégias de abordagens, as formas de prevenção que poderiam ser mais efetivas conforme os grupos, alguns membros inclusive se disponibilizaram e foram algumas vezes com o Bonde para as ações de prevenção.

Ocorriam em espaços distintos como ruas de diversos bairros, escolas secundaristas, escolas de nível superior. O público era abordado em diferentes circunstâncias enquanto transitavam, aguardavam transporte, se deslocavam entre as salas de aula, lanchavam, participavam de festas populares. Diversas pessoas foram incluídas nas ações entre elas estudantes, trabalhadores formais e informais e pessoas que se encontravam em situação de rua.

A aproximação era sempre iniciada com a apresentação pessoal, seguida de informações sobre o Bonde e os objetivos da atividade. Esse momento foi marcado por sentimento de insegurança, medo e anseio para maioria das graduandas que faziam parte da equipe executora. Muitas nunca haviam realizado ações de extensão, assim a experiência nova, o contato com pessoas desconhecidas que poderiam ser receptivas ou não as abordagens, o falar sobre uma temática ainda tabu pela sociedade foram algumas das situações que acarretaram desconforto.

As reações das pessoas convidadas a participarem das atividades foram múltiplas, a exemplo de movimentos de esquiva e linguagens corporais que evidenciavam a recusa, diante destes movimentos a abordagem era interrompida. Apesar disso, foram evidenciadas expressões de aceitação, acolhimento e curiosidade.

Os sujeitos evidenciaram a singularidade e subjetividade que lhes são peculiares, as experiências vividas permitiram o reconhecimento da condição de vulnerabilidade como princípio fundamental para compreensão da transmissibilidade das IST. Para ampliar o acesso a prevenção o uso da linguagem horizontal, do cuidado empático e integral capaz de minimizar exposição e aproximar os sujeitos de práticas sexuais seguras. 
Nas ações a disponibilização dos preservativos e gel lubrificantes era um momento muito aguardado, que gerava, às vezes, disputa entre os participantes. Deste modo, tivemos iniciativa de solicitar a autorização das instituições de ensino para instalar dispensadores de insumos nos banheiros. $O$ abastecimento desse material era feito semanalmente, embora tenhamos sido avisadas pelos estudantes que a quantidade não atendia a demanda deles. Essa medida facilitou o acesso aos dispositivos de prevenção visto que eles referiram não ir buscá-los nas unidades de saúde.

Ainda entre os estudantes, outra medida de impacto foi a testagem para o HIV com o fluido oral, esta teve o apoio de uma Instituição Beneficente que treinou a equipe, forneceu os testes, acompanhou o aconselhamento pré e pós teste e a testagem, acolhendo e fazendo o encaminhamento dos estudantes com sorologia positiva. A realização de testagens sorológicas para o HIV e confronto com resultados positivos suscitou sensações de aflição em algumas integrantes da equipe executora. Todavia, a presença da docente e dos profissionais acompanhando as atividades foi fundamental para fortalecer e assegurar uma conduta adequada, não interferindo no processo de acolhimento, escuta qualificada e encaminhamento dessas pessoas.

A articulação do Bonde com a Secretaria Municipal de Saúde de Salvador foi importante, pois a mesma disponibilizou preservativos vaginais (interno) e penianos (externo), gel lubrificante, materiais educativos que falavam sobre as IST, as Profilaxias Pré e Pós-exposição (PREP e PEP), os serviços que disponibilizavam o atendimento, além de cartilhas que orientavam a prevenção, a Rede de Atenção a Saúde do Município, além do processo de treinamento para testagem sorológica para o HIV com fluido oral, ofertado para o grupo que participou de abordagens na rua sob supervisão, como forma de exercício.

O grupo de apoio e acolhimento de pessoas convivendo com o HIV/Aids nos ajudou muito por permitir a aproximação com um universo até então desconhecido, o lugar de fala de pessoas que convivem com o HIV. Eles discutiam as estratégias de abordagens, as formas de prevenção que poderiam ser mais efetivas conforme os grupos, alguns membros inclusive se disponibilizaram e foram algumas vezes com o Bonde para as ações de prevenção.

Por conseguinte, deu-se início ao planejamento das ações extensionistas. Nesta etapa, foram realizadas reuniões semanais, discussões e estudos sobre os temas fomentando a construção das estratégias a serem utilizadas em cada ação.

As ações extensionistas foram realizadas no período de junho de 2018 a dezembro de 2019, respeitando o sigilo da identidade dos participantes das atividades, a ética, a fim de proteger a intimidade das pessoas e/ou grupos envolvidos.

\section{DISCUSSÃO}

Diante da receptividade em dialogar sobre os assuntos, e da aceitação da população participante, inúmeras possibilidades estavam à disposição para serem utilizadas durantes as ações. Segundo Pinto CL e Tavares HM (2010), o lúdico é algo fundamental no processo de aprendizagem, pois resgata as vivências, valores, crenças e conhecimentos de cada sujeito tornando-o componente na construção de sua aprendizagem. Por este motivo, foram utilizadas algumas atividades lúdicas para estimular as discussões e a sensibilização das pessoas.

Dentre essas atividades, a confecção de uma caixa surpresa intitulada: "Meu amigo quer saber", continha perguntas a respeito das IST e uma questão era sorteada pelas pessoas que participavam, sendo este 0 disparador para o início das discussões. Os jogos educativos favorecem o desenvolvimento da educação estimulando o avanço do conhecimento em saúde (YONEKURA T e SOARES CB, 2010).

Emergiram algumas curiosidades sobre sintomatologias de algumas IST, visto que nem todas apresentam sinais/sintomas, e desta forma algumas queixas ginecológicas estão associadas a estas infecções. No entanto, foi salientada, a necessidade de buscarem um serviço de saúde para uma melhor investigação, possível diagnóstico e tratamento. Destacamos a gratuidade do tratamento no SUS, a atenção básica como principal responsável em acolher essa demanda, estando assim as unidades de saúde preparadas para atender a população com IST (BRASIL, 2019). 
Nas atividades percebemos a perspectiva de invulnerabilidade de muitas pessoas, pois diante da disponibilização dos preservativos referiam não precisar dos insumos por serem fiéis a parceria, casados (as), evangélicos (as), entre outras. Cabe salientar, que esse discurso, é reflexo dos estigmas que acompanham a história do HIV/Aids desde os seus primórdios, visto que foi vírus foi justificado por alguns seguimentos religiosos como fruto do pecado, o que só reforçou os estereótipos em torno das pessoas consideradas no início da epidemia como "grupo de risco" (ESPÍRITO SANTO CC e GOMES AM, 2017).

Muitas mulheres ressaltaram em suas falas que ao retornarem a suas residências com os preservativos disponibilizados durante as ações poderiam ser acusadas de infiéis por seus companheiros, o que nos leva a reflexão do quão enraizado está o machismo na sociedade. Elas encontram-se em condições de vulnerabilidade em múltiplos contextos, no que se refere às infecções sexualmente transmissíveis, em virtude de uma violência estrutural, que enfatiza que a inserção de preservativos nas práticas heterossexuais pode deduzir a ocorrência de infidelidade e desconfiança tornando-as mais suscetíveis a aquisição de IST (SILVA CM e VARGENS OM, 2009).

Contudo, durante as abordagens com os estudantes, tanto de nível médio quanto de nível superior percebemos a curiosidade pelos diversos temas abordados. Levávamos modelos anatômicos do órgão sexual masculino e feminino e orientávamos a colocação das camisinhas, do gel lubrificante. Dúvidas a respeito do uso correto da camisinha vaginal (interna) eram muito frequentes. Este preservativo foi lançado no Brasil em 1997, porém sua aceitação no mercado não tem sido tão efetiva por conta do preço mais elevado quando comparada ao preservativo peniano (externa), pela dificuldade de encontrar nas Unidade Básica de Saúde (UBS), já que as mesmas não são produzidas em grande escala, e desta forma nem todas as pessoas têm acesso. (BRASIL, 2019).

De acordo com Silva IT, et. al (2017), faz-se necessária a ampliação da testagem sorológica e capacitação de profissionais da equipe de saúde que atuam na estratégia de Saúde da Família para oferta de um atendimento de qualidade e efetivo, as unidades precisam estar preparadas para favorecer o acesso oportuno e precoce ao diagnóstico, possibilitando tratamento precoce e consequentemente a melhoria da qualidade de vida..

Além disso, o embasamento teórico se configurou como parte fundamental nas ações, uma vez qu eiríamos abordar pessoas que poderiam não apresentar sintomas, nem ter queixas, mas que poderiam estar infectadas e ter a saúde comprometida (SILVA MJ; SCHRAIBER LB e MOTA A, 2019). Atrelado a isso, havia a possibilidade de encontrar pessoas que apresentariam diversas demandas de saúde e outras que sequer reconheciam as suas reais necessidades, entendendo a subjetividade que envolve a necessidade e que muitas vezes não são reconhecidas (SOUZA D e IRIART J, 2018).

A partir das leituras o nosso espectro de visão foi ampliado e novas estratégias de aprendizado foram surgindo como as rodas de conversa sobre gênero, raça e classe. Identificamos que cada sujeito se percebia e percebia o outro de diferentes formas, questões como experiências de vida, contextos e marcadores sociais influenciam a maneira de ser e viver de cada um. Então, a saúde, os hábitos de vida e comportamentos sofrem múltiplas influências (SILVA VC e SILVA WS, 2018). O estudo das vulnerabilidades e suas amplas dimensões foram pautas das reuniões do grupo, visto que este conceito é elaborado a partir da tentativa de explicar a chance de exposição das pessoas ao vírus HIV e sua compreensão se dá a partir de perspectivas individuais, sociais e programáticas (AYRES JR et.al, 2009).

Assim, são reconhecidas as diferentes vulnerabilidades das crianças, jovens, mulheres e idosos. As mulheres, em especial, estão condicionadas há um lugar de subalternidade frente aos homens, a estrutura patriarcal de cunho machista estabelecida na sociedade contribui para que as mulheres estejam mais suscetíveis a violência, às IST, pois sofrem a opressão que violenta seus corpos, ceifando a autonomia, o poder de negociação para manutenção de práticas sexuais seguras (BRASIL, 2007).

A articulação entre conceitos basilares foi uma ferramenta chave para subsidiar a interlocução presente na organização e execução das ações, a interseccionalidade cunhada por Crenshaw K (2002), propiciou a análise de como os marcadores de gênero, raça e classe dialogam e se sobrepõem, e desta forma reverberam na vida de cada sujeito de maneira distinta. 
Esclareceram dúvidas e apresentaram práticas exitosas de atendimento, segundo o protocolo do Ministério da Saúde (BRASIL, 2019). Houve também a aproximação com a Rede de Atenção à Saúde do Município, foram elencadas as instâncias que podem ser acionadas para o enfrentamento da violência, a exemplo da lei 11.340/06 que trata das medidas de prevenção e combate, bem como, toda a rede de apoio incluindo a Casa Abrigo, a delegacia e entre outras (BRASIL, 2006).

Ações educativas que visem à sensibilização das pessoas a adotarem práticas sexuais seguras são fundamentais, sobretudo, às mulheres que estão imersas a contextos de vulnerabilidade. Nesse sentido, a participação do Bonde contribuiu no amadurecimento pessoal, nas vivências de múltiplas situações novas, no aprofundamento teórico e prático, além do exercício de um papel social como agente de mudança, apesar da carga horária do curso de enfermagem se configurar como um dificultador em alguns momentos, viabilizou também o uso de Metodologias Ativas como ferramenta de aproximação com a comunidade. Logo, as atividades extensionistas além de promoverem a formação acadêmica, proporcionam o fortalecimento de vínculos entre academia e comunidade.

\section{REFERÊNCIAS}

1. AYRES JRCM, et. al. Risco, vulnerabilidade e práticas de prevenção e promoção da saúde. In:Tratado de saúde coletiva [S.I: s.n.], 2009.

2. BRASIL. Ministério da Saúde. Plano Integrado de enfrentamento da feminização da epidemia de AIDS e outras DST. Secretaria de Vigilância em saúde. Programa Nacional da DST/Aids, 2007.

3. Brasil. Ministério da Saúde. Secretaria de Vigilância em Saúde. Departamento de Vigilância, Prevenção e Controle das Infecções Sexualmente Transmissíveis, do HIV/Aids e das Hepatites Virais. Prevenção Combinada do HIV/Bases conceituais para profissionais, trabalhadores(as) e gestores(as) de saúde/Ministério da Saúde, Secretaria de Vigilância em Saúde, Departamento de Vigilância, Prevenção e Controle das Infecções Sexualmente Transmissíveis, do HIV/Aids e das Hepatites Virais. - Brasília: Ministério da Saúde, 2017.

4. Ministério da Saúde. Protocolo Clínico e diretrizes terapêuticas para atenção integral às pessoas com infecções sexualmente transmissíveis (IST). Brasília-DF, 2019.

5. Lei Maria da Penha. Lei no 11.340/ 2006. Coíbe a violência doméstica e familiar contra a mulher. Presidência da República, 2006.

6. CRENSHAW KW. A interseccionalidade na discriminação de raça e gênero. In: VV.AA. Cruzamento: raça e gênero, Brasília: Unifem, 2004.

7. ESPÍRITO-SANTO CC, GOMES AMT. O pensamento religioso no contexto da sexualidade e saúde. In: LIMA CF et al. Sexualidades e saúde: perspectivas para um cuidado ampliado. 1a ed. Rio de Janeiro: Bonecker Editora, 2017. p.59-77.

8. FREIRE P. A pedagogia do oprimido. Rio de Janeiro, paz e terra, 1987.

9. PASSOS TS, HORA AB, PAIXÃO ALSS da et al. Educação em saúde para prevenção de infecções sexualmente transmissíveis em comunidades quilombolas. Rev enferm UFPE on line., Recife, 11(10):3965-70, out., 2017

10. PINTO CL, TAVARES HM. O Lúdico na Aprendizagem: Aprender a Aprender. Revista da Católica, Uberlândia, 2010; v. 2, n. 3, p. 226-235.

11. SILVA CM, VARGENS OMC. A percepção de mulheres quanto à vulnerabilidade feminina para contrair DST/HIV. Revista da Escola de Enfermagem da USP, São Paulo, 2009; 43, n. 2, p. 401-406.

12. SILVA ITS et al. Cartografia da implementação do teste rápido anti-HIV na Estratégia Saúde da Família: perspectiva de enfermeiros. Esc Anna Nery, 2017; 21(4):e20170019.

13. SILVA MJS et al. O conceito de saúde na Saúde Coletiva: contribuições a partir da crítica social e histórica da produção científica. Physis, Rio de Janeiro, 2019; v.29, n. 1, e290102.

14. SILVA VC, SILVA WS. Marcadores sociais da diferença: uma perspectiva interseccional sobre ser estudante negro e deficiente no Ensino Superior brasileiro. Revista Educação Especial, 2018; v. 31, n. 62, p. 569-586.

15. SOUSA D, IRIART J. "Viver dignamente": necessidades e demandas de saúde de homens trans em Salvador, Bahia, Brasil. Cad. Saúde Pública, Rio de Janeiro, 2018; v. 34, n. 10, e00036318.

16. YONEKURA T, SOARES CB. O jogo educativo como estratégia de sensibilização para coleta de dados com adolescentes. Rev. Latino-Am. Enfermagem, 2010;18(5):[07 telas]. 